\title{
Integral cohomology and Chern classes of the special linear group over the ring of integers
}

\author{
By DOMINIQUE ARLETTAZ \\ Institut de mathématiques, Université de Lausanne, 1015 Lausanne, Switzerland \\ e-mail: dominique.arlettaz@ima.unil.ch \\ CHRISTIAN AUSONI \\ Departement Mathematik, HG, ETH-Zentrum, 8092 Zürich, Switzerland \\ e-mail: ausoni@math.ethz.ch \\ MAMORU MIMURA \\ Department of Mathematics, Faculty of Science, Okayama University, Okayama, Japan 700 \\ e-mail: mimura@math.okayama-u.ac.jp \\ and NOBUAKI YAGITA \\ Faculty of Education, Ibaraki University, Mito, Ibaraki, Japan \\ e-mail: yagita@mito.ipc.ibaraki.ac.jp
}

\begin{abstract}
This paper is devoted to the complete calculation of the additive structure of the 2-torsion of the integral cohomology of the infinite special linear group $S L(\mathbb{Z})$ over the ring of integers $\mathbb{Z}$. This enables us to determine the best upper bound for the order of the Chern classes of all integral and rational representations of discrete groups.
\end{abstract}

\section{Introduction}

The Hopf algebra structure of the mod 2 cohomology of the infinite special and general linear groups $S L(\mathbb{Z})$ and $G L(\mathbb{Z})$ over the ring of integers $\mathbb{Z}$ has been completely determined in [AMNY] as a module over the Steenrod algebra. For instance, $H^{*}(S L(\mathbb{Z}) ; \mathbb{Z} / 2) \cong H^{*}\left(B S L(\mathbb{Z})^{+} ; \mathbb{Z} / 2\right)$ is the tensor product of a polynomial algebra with an exterior algebra:

$$
\begin{aligned}
H^{*}\left(B S L(\mathbb{Z})^{+} ; \mathbb{Z} / 2\right) & \cong H^{*}(B S O ; \mathbb{Z} / 2) \otimes H^{*}(S U ; \mathbb{Z} / 2) \\
& \cong \mathbb{Z} / 2\left[w_{2}, w_{3}, \ldots, w_{j}, \ldots\right] \otimes \Lambda_{\mathbb{Z} / 2}\left(u_{3}, u_{5}, \ldots, u_{2 k-1}, \ldots\right),
\end{aligned}
$$

where $\operatorname{deg}\left(w_{j}\right)=j$ and $\operatorname{deg}\left(u_{2 k-1}\right)=2 k-1$.

The first goal of this paper is to investigate the mod 2 cohomological Bockstein spectral sequence

$$
E_{1}^{*}\left(B S L(\mathbb{Z})^{+}\right) \cong H^{*}\left(B S L(\mathbb{Z})^{+} ; \mathbb{Z} / 2\right) \Longrightarrow\left(H^{*}\left(B S L(\mathbb{Z})^{+} ; \mathbb{Z}\right) / \text { torsion }\right) \otimes \mathbb{Z} / 2
$$

2000 Mathematics Subject Classification: Primary 20 G 10; Secondary 19 D 55, 55 R 40, 55 T 99, 57 R 20. 
of the space $B S L(\mathbb{Z})^{+}$(see Sections $1-5$ of $[\mathrm{Brd}]$ ). By using the $\bmod 2$ Bockstein spectral sequences of the spaces $B S O$ and $B S L\left(\mathbb{F}_{p}\right)^{+}$(for a prime $p \equiv 5 \bmod 8$ ) and the maps $h: B S L(\mathbb{Z})^{+} \rightarrow B S O$ and $f_{p}: B S L(\mathbb{Z})^{+} \rightarrow B S L\left(\mathbb{F}_{p}\right)^{+}$induced by the inclusion $\mathbb{Z} \hookrightarrow \mathbb{R}$ and by the reduction $\bmod p$ respectively, we compute the terms $E_{r}^{*}\left(B S L(\mathbb{Z})^{+}\right)$and the differentials $d_{r}$ for all $r \geq 1$ (see Theorem 4.3 and Corollary 4.4). Of course, this detects the 2 -torsion of the integral cohomology $H^{*}\left(B S L(\mathbb{Z})^{+} ; \mathbb{Z}\right) \cong H^{*}(S L(\mathbb{Z}) ; \mathbb{Z})$ of the special linear group $S L(\mathbb{Z})$. Theorem 4.7 actually provides an explicit additive presentation of the 2-torsion of $H^{*}\left(B S L(\mathbb{Z})^{+} ; \mathbb{Z}\right)$ by generators and relations (see also Remark 4.8 for some partial information on the multiplicative structure). It turns out that $H^{*}\left(B S L(\mathbb{Z})^{+} ; \mathbb{Z}\right)$ contains no cyclic direct summand of order 4 and that the set of all non-trivial elements of $\Lambda_{\mathbb{Z} / 2}\left(u_{4 i+1}, i \geq 1\right)$ is in one-to-one correspondence with an additive basis of $H^{*}\left(B S L(\mathbb{Z})^{+} ; \mathbb{Z}\right) /$ torsion (see Corollary 4.6). Moreover, we are able to understand the effect of the induced homomorphisms $h^{*}: H^{*}(B S O ; \mathbb{Z}) \rightarrow H^{*}\left(B S L(\mathbb{Z})^{+} ; \mathbb{Z}\right)$ and $f_{p}^{*}: H^{*}\left(B S L\left(\mathbb{F}_{p}\right)^{+} ; \mathbb{Z}\right) \rightarrow$ $H^{*}\left(B S L(\mathbb{Z})^{+}, \mathbb{Z}\right)$ on the 2-torsion elements: Theorem 4.9 asserts in particular that $h^{*}$ is injective on the elements of order 2 and that $f_{p}^{*}$ is injective on all cyclic direct summands of order $2^{r}$ with $r \geq 3$.

Notice that it is easy to extend these results to the integral cohomology $H^{*}(G L(\mathbb{Z}) ; \mathbb{Z}) \cong H^{*}\left(B G L(\mathbb{Z})^{+} ; \mathbb{Z}\right)$ of the general linear group $G L(\mathbb{Z})$ because of the homotopy equivalence $B G L(\mathbb{Z})^{+} \simeq B S L(\mathbb{Z})^{+} \times B \mathbb{Z} / 2$ (see for example [Ar1], Lemma 1.2).

As a consequence, we obtain the exact order of all Chern classes $c_{n}(S L(\mathbb{Z})) \in H^{2 n}(S L(\mathbb{Z}) ; \mathbb{Z})$ of the inclusion $S L(\mathbb{Z}) \hookrightarrow G L(\mathbb{C})$ (see Proposition 5.2 and Theorem 5.3) and deduce the best upper bound for the order of the Chern classes of all integral and rational representations of discrete groups (see Corollary 5.6).

The paper is organized as follows. Sections 2 and 3 present the mod 2 Bockstein spectral sequence for the spaces $B S O$ and $B S L\left(\mathbb{F}_{p}\right)^{+}$respectively. The mod 2 Bockstein spectral sequence and the 2-torsion of the integral cohomology of $B S L(\mathbb{Z})^{+}$are computed in Section 4. Finally, Section 5 is devoted to the investigation of the order of the Chern classes of integral and rational representations of discrete groups.

\section{The mod 2 Bockstein spectral sequence for $B S O$}

The determination of $H^{*}\left(B S L(\mathbb{Z})^{+} ; \mathbb{Z} / 2\right)$ is based on cohomological calculations involving the pull-back diagram

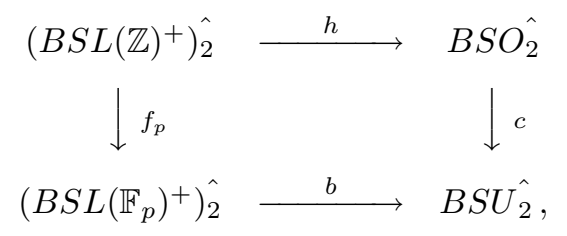

where $\left(-\hat{2}_{2}\right.$ denotes the completion at the prime $2, p$ any prime $\equiv 3$ or $5 \bmod 8, h$ the map induced by the inclusion $\mathbb{Z} \hookrightarrow \mathbb{R}, f_{p}$ the map induced by the reduction $\bmod p: \mathbb{Z} \rightarrow \mathbb{F}_{p}, c$ the complexification and $b$ the Brauer lift, and where the homotopy fibers of both horizontal maps are $S U_{2}$ (for the details of that construction, see $[\mathrm{AMNY}]$, where the argument is presented for $G L(\mathbb{Z})$ instead of $S L(\mathbb{Z})$, or Chapter 3 of $[\mathrm{Au}]$; notice also that an unstable version of this computation is given in [He]). In order to go through the mod 2 Bockstein spectral sequence for $B S L(\mathbb{Z})^{+}$, we shall first consider the mod 2 Bockstein spectral sequence for the spaces $B S O$ and $B S L\left(\mathbb{F}_{p}\right)^{+}$, and the homomorphisms induced in cohomology by the maps $h$ and $f_{p}$. 
Let us start by looking at the mod 2 Bockstein spectral sequence

$$
E_{1}^{*}(B S O) \cong H^{*}(B S O ; \mathbb{Z} / 2) \cong \mathbb{Z} / 2\left[w_{2}, w_{3}, \ldots, w_{j}, \ldots\right] \Longrightarrow\left(H^{*}(B S O ; \mathbb{Z}) / \text { torsion }\right) \otimes \mathbb{Z} / 2 .
$$

Its first differential is $d_{1}=S q^{1}$ and we know by Wu's formula (see for instance [MT], Part I, p.138, Theorem 5.12 ) that for $i \geq 1, S q^{1}\left(w_{2 i}\right)=w_{2 i+1}, S q^{1}\left(w_{2 i+1}\right)=0$, and that $S q^{1}\left(w_{j}^{2}\right)=0$ for $j \geq 1$. Thus, we may deduce that

$$
E_{2}^{*}(B S O) \cong \mathbb{Z} / 2\left[w_{2}^{2}, w_{4}^{2}, \ldots, w_{2 i}^{2}, \ldots\right]
$$

is concentrated in degrees $\equiv 0 \bmod 4$. Since $d_{r}$ is of degree 1 , it is then obvious that $d_{r}=0$ for all $r \geq 2$. Consequently, we have proved the following result.

\section{Proposition 2.1.}

(a) The mod 2 Bockstein spectral sequence for $B S O$ has the property that $E_{1}^{*}(B S O) \cong \mathbb{Z} / 2\left[w_{j}, j \geq 2\right]$ and $E_{r}^{*}(B S O) \cong E_{\infty}^{*}(B S O) \cong \mathbb{Z} / 2\left[w_{2 i}^{2}, i \geq 1\right]$ for all $r \geq 2$.

(b) All non-trivial 2-torsion elements of $H^{*}(B S O ; \mathbb{Z})$ have order exactly equal to 2 .

(c) $H^{*}(B S O ; \mathbb{Z}) /$ torsion $\cong \mathbb{Z}\left[p_{4 i}, i \geq 1\right]$, where $p_{4 i}$ is of degree $4 i$ and represents an element of $H^{4 i}(B S O ; \mathbb{Z})$ whose reduction $\bmod 2$ is $w_{2 i}^{2} \in H^{4 i}(B S O ; \mathbb{Z} / 2)$.

Remark 2.2. The additive and multiplicative structures of the 2 -torsion of $H^{*}(B S O ; \mathbb{Z})$ has been obtained a long time ago in [Brn], Theorem 1.5, and [F], Theorem 1 (see also [Bo], Theorem 24.7 and Proposition 25.6, $[\mathrm{CV}]$, Theorem 1, and [ThE], Theorem A). For completeness, let us recall here its additive structure, which can also be determined by the argument we shall use in the next sections (see Lemma 3.6 and Theorems 3.7 and 4.7): if $\Psi$ denotes the graded $\mathbb{Z}$-algebra $\mathbb{Z}\left[q_{2 i+1}, i \geq 1\right] \otimes \mathbb{Z}\left[p_{4 i}, i \geq 1\right]$ with $\operatorname{deg}\left(q_{2 i+1}\right)=2 i+1$ and $\operatorname{deg}\left(p_{4 i}\right)=4 i$, then the 2 -torsion subgroup of $H^{*}(B S O ; \mathbb{Z})$ is additively isomorphic to the graded $\Psi$-module generated by

$$
\left\{G_{A} \mid A \text { running over all non-empty finite subsets of } N_{1}=\mathbb{N}-\{0\}\right\}
$$

with relations generated by

$$
\left\{2 G_{A}, \sum_{i \in A} q_{2 i+1} G_{A-\{i\}}\right\} .
$$

Here, the element $G_{\left\{i_{1}, \ldots, i_{t}\right\}} \in H^{*}(B S O ; \mathbb{Z})$ is of degree $2\left(\sum_{j=1}^{t} i_{j}\right)+1$ and reduces mod 2 to the class $\sum_{j=1}^{t} w_{2 i_{j}+1} w_{2 i_{1}} \cdots w_{2 i_{(j-1)}} w_{2 i_{(j+1)}} \cdots w_{2 i_{t}} \in H^{*}(B S O ; \mathbb{Z} / 2)$.

\section{The mod 2 Bockstein spectral sequence for $B S L\left(\mathbb{F}_{p}\right)^{+}$}

In this section, let us consider the space $B S L\left(\mathbb{F}_{p}\right)^{+}$for any prime number $p \equiv 5 \bmod 8$. Its mod 2 cohomology is $H^{*}\left(B S L\left(\mathbb{F}_{p}\right)^{+} ; \mathbb{Z} / 2\right) \cong \mathbb{Z} / 2\left[c_{2}, c_{3}, \ldots, c_{k}, \ldots\right] \otimes \Lambda_{\mathbb{Z} / 2}\left(e_{2}, e_{3}, \ldots, e_{k}, \ldots\right)$ with $\operatorname{deg}\left(c_{k}\right)=2 k$ and $\operatorname{deg}\left(e_{k}\right)=2 k-1$ (see Theorem 1 of $[\mathrm{Q}]$ ). The first differential of its mod 2 Bockstein spectral sequence

$$
E_{1}^{*}\left(B S L\left(\mathbb{F}_{p}\right)^{+}\right) \cong \mathbb{Z} / 2\left[c_{k}, k \geq 2\right] \otimes \Lambda_{\mathbb{Z} / 2}\left(e_{k}, k \geq 2\right) \Longrightarrow\left(H^{*}\left(B S L\left(\mathbb{F}_{p}\right)^{+} ; \mathbb{Z}\right) / \text { torsion }\right) \otimes \mathbb{Z} / 2
$$

is trivial since $d_{1}\left(c_{k}\right)=S q^{1}\left(c_{k}\right)=0$ and $d_{1}\left(e_{k}\right)=S q^{1}\left(e_{k}\right)=0$ for all $k \geq 2$ according to Lemmas 3 and 4 of [Ar4]. Thus,

$$
E_{2}^{*}\left(B S L\left(\mathbb{F}_{p}\right)^{+}\right) \cong E_{1}^{*}\left(B S L\left(\mathbb{F}_{p}\right)^{+}\right) .
$$

In order to understand the higher differentials $d_{r}$, let us recall the definition of $d_{r}$ (see Section 1 of [Brd]). If $x \in E_{r}^{n}\left(B S L\left(\mathbb{F}_{p}\right)^{+}\right)$, then there is an element $\widetilde{x} \in H^{n}\left(B S L\left(\mathbb{F}_{p}\right)^{+} ; \mathbb{Z} / 2^{r}\right)$ such that the homomorphism $\theta_{r}: H^{n}\left(B S L\left(\mathbb{F}_{p}\right)^{+} ; \mathbb{Z} / 2^{r}\right) \rightarrow H^{n}\left(B S L\left(\mathbb{F}_{p}\right)^{+} ; \mathbb{Z} / 2\right)$ induced by the natural surjection $\mathbb{Z} / 2^{r} \rightarrow \mathbb{Z} / 2$ sends 
$\widetilde{x}$ onto $x$. Let $\beta_{r}: H^{n}\left(B S L\left(\mathbb{F}_{p}\right)^{+} ; \mathbb{Z} / 2^{r}\right) \rightarrow H^{n+1}\left(B S L\left(\mathbb{F}_{p}\right)^{+} ; \mathbb{Z}\right)$ denote the Bockstein homomorphism associated with the short exact sequence

$$
0 \longrightarrow \mathbb{Z} \stackrel{\cdot 2^{r}}{\longrightarrow} \mathbb{Z} \longrightarrow \mathbb{Z} / 2^{r} \longrightarrow 0
$$

and $\operatorname{red}_{2}: H^{n+1}\left(B S L\left(\mathbb{F}_{p}\right)^{+} ; \mathbb{Z}\right) \rightarrow H^{n+1}\left(B S L\left(\mathbb{F}_{p}\right)^{+} ; \mathbb{Z} / 2\right)$ the reduction $\bmod 2$. Then, the differential $d_{r}: E_{r}^{n}\left(B S L\left(\mathbb{F}_{p}\right)^{+}\right) \rightarrow E_{r}^{n+1}\left(B S L\left(\mathbb{F}_{p}\right)^{+}\right)$is defined by

$$
d_{r}(x)=\operatorname{red}_{2}\left(\beta_{r}(\widetilde{x})\right) .
$$

Let us apply this to the case of the space $B S L\left(\mathbb{F}_{p}\right)^{+}$.

Definition 3.1. For any integer $r \geq 2$, let $N_{r}=\left\{k \in \mathbb{N} \mid v_{2}(k)=r-2\right\}$, where $v_{2}(-)$ is the 2-adic valuation (in other words, $N_{r}=\left\{k=2^{r-1} i+2^{r-2} \mid i \geq 0\right\}$ ).

Remark 3.2. For any prime $p \equiv 5 \bmod 8$ and any integer $r \geq 2, N_{r}=\left\{k \in \mathbb{N} \mid v_{2}\left(p^{k}-1\right)=r\right\}$. In order to check this, it is sufficient to show that $v_{2}\left(p^{k}-1\right)=v_{2}(k)+2$ for any positive integer $k$. Let us write $p=4 m+1$ with $m$ odd. Then $p^{k}-1=\sum_{t=1}^{k}\left(\begin{array}{l}k \\ t\end{array}\right) 4^{t} m^{t}$. For $t \geq 2$, one has

$$
v_{2}\left(\left(\begin{array}{l}
k \\
t
\end{array}\right) 4^{t} m^{t}\right)=2 t+v_{2}\left(\frac{k(k-1) \cdots(k-t+1)}{t !}\right) \geq 2 t+v_{2}(k)-v_{2}(t !) \geq v_{2}(k)+t+1 \geq v_{2}(k)+3,
$$

since $v_{2}(t !) \leq t-1$. This implies that $v_{2}\left(p^{k}-1\right)=v_{2}(4 k m)=v_{2}(k)+2$.

Lemma 3.3. Let $p$ be any prime $\equiv 5 \bmod 8$ and $r$ be any integer $\geq 2$. If $k \in N_{r}$, then the class $e_{k}$ belongs to $E_{s}^{2 k-1}\left(B S L\left(\mathbb{F}_{p}\right)^{+}\right)$for all $s \leq r$ and $d_{r}\left(e_{k}\right)=c_{k} \in E_{r}^{2 k}\left(B S L\left(\mathbb{F}_{p}\right)^{+}\right)$.

Proof. If $k$ belongs to $N_{r}$, then $r=v_{2}\left(p^{k}-1\right)$ by Remark 3.2. Thus, according to Section 3 of [Q], $e_{k}$ is the image of an element $\widetilde{e}_{k} \in H^{2 k-1}\left(B S L\left(\mathbb{F}_{p}\right)^{+} ; \mathbb{Z} / 2^{r}\right)$ under the homomorphism $\theta_{r}$ and consequently, $e_{k} \in E_{r}^{2 k-1}\left(B S L\left(\mathbb{F}_{p}\right)^{+}\right)$. Moreover, it follows from Lemma 5 of $[\mathrm{Q}]$ that $d_{r}\left(e_{k}\right)=\operatorname{red}_{2}\left(\beta_{r}\left(\widetilde{e}_{k}\right)\right)=c_{k}$.

We get the complete calculation of the mod 2 Bockstein spectral sequence for the space $B S L\left(\mathbb{F}_{p}\right)^{+}$.

Theorem 3.4. For any prime $p \equiv 5 \bmod 8$, the $\bmod 2$ Bockstein spectral sequence for $B S L\left(\mathbb{F}_{p}\right)^{+}$satisfies:

(a) $E_{2}^{*}\left(B S L\left(\mathbb{F}_{p}\right)^{+}\right) \cong E_{1}^{*}\left(B S L\left(\mathbb{F}_{p}\right)^{+}\right) \cong \mathbb{Z} / 2\left[c_{k}, k \geq 2\right] \otimes \Lambda_{\mathbb{Z} / 2}\left(e_{k}, k \geq 2\right)$ and $d_{2}\left(e_{k}\right)=c_{k}$ whenever $k \in N_{2}=\{$ odd positive integers $\}$

(b) for any $r \geq 3, E_{r}^{*}\left(B S L\left(\mathbb{F}_{p}\right)^{+}\right) \cong \mathbb{Z} / 2\left[c_{k}, k \in N_{s}\right.$ for $\left.s \geq r\right] \otimes \Lambda_{\mathbb{Z} / 2}\left(e_{k}, k \in N_{s}\right.$ for $s \geq r$ ) and $d_{r}\left(e_{k}\right)=c_{k}$ whenever $k \in N_{r}$,

(c) $E_{\infty}^{*}\left(B S L\left(\mathbb{F}_{p}\right)^{+}\right)=0$.

By looking at the differential graded $\mathbb{Z} / 2$-algebras

$$
F_{s}=\mathbb{Z} / 2\left[c_{k}, 2 \leq k \in N_{s}\right] \otimes \Lambda_{\mathbb{Z} / 2}\left(e_{k}, 2 \leq k \in N_{s}\right), \quad \delta_{s}\left(e_{k}\right)=c_{k}, \quad \text { for } s \geq 2,
$$

one can write the $E_{r}$-terms of the mod 2 Bockstein spectral sequence of $B S L\left(\mathbb{F}_{p}\right)^{+}$as follows.

Corollary 3.5. For any prime $p \equiv 5 \bmod 8$ and for any integer $r \geq 2, E_{r}^{*}\left(B S L\left(\mathbb{F}_{p}\right)^{+}\right) \cong \bigotimes_{s \geq r} F_{s}$ with the differential $d_{r}=\delta_{r}$ on $F_{r}$ and $d_{r}=0$ on $F_{s}$ when $s>r$.

The knowledge of the mod 2 Bockstein spectral sequence determines the additive structure of the 2-torsion of the integral cohomology $H^{*}\left(B S L\left(\mathbb{F}_{p}\right)^{+} ; \mathbb{Z}\right)$ since the elements of the image of $d_{r}$ detect the elements of order $2^{r}$ in $H^{*}\left(B S L\left(\mathbb{F}_{p}\right)^{+} ; \mathbb{Z}\right)$. Let us start with the following observation. 
Lemma 3.6. Consider a set $N$ and the differential graded $\mathbb{Z} / 2$-algebra

$$
D_{N}=\mathbb{Z} / 2\left[x_{n}, n \in N\right] \otimes \Lambda_{\mathbb{Z} / 2}\left(y_{n}, n \in N\right),
$$

where the differential is a derivation $\delta$ given by $\delta\left(y_{n}\right)=x_{n}$. Then the image of $\delta$ is the $\mathbb{Z} / 2\left[x_{n}, n \in N\right]$ module generated by

$$
\left\{H_{A}=\sum_{a \in A} x_{a} \prod_{b \in A-\{a\}} y_{b} \mid A \text { running over all non-empty finite subsets of } N\right\}
$$

with the relations generated by

$$
\left\{\sum_{a \in A} x_{a} H_{A-\{a\}} \mid A \text { running over all non-empty finite subsets of } N\right\} \text {. }
$$

Proof. Let us write $P$ for the polynomial tensor factor $\mathbb{Z} / 2\left[x_{n}, n \in N\right]$ of $D_{N}$. Since $\delta^{2}=0$, one has $\delta(P)=0$ and the fact that $\delta$ is a derivation shows that $D_{N} \rightarrow \operatorname{Im}(\delta)$ is a morphism of $P$-modules. The elements of the form $\prod_{a \in A} y_{a}$, where $A$ runs over all non-empty finite subsets of $N$, generate $D_{N}$ as a $P$-module. Therefore, the image of $\delta$ is generated, as a $P$-module, by the elements $H_{A}=\delta\left(\prod_{a \in A} y_{a}\right)$. We get obviously the relations $\sum_{a \in A} x_{a} H_{A-\{a\}}=\delta^{2}\left(\prod_{a \in A} y_{a}\right)=0$ and there are no other relations because $H_{*}\left(D_{N}, \delta\right) \cong \mathbb{Z} / 2$.

Let us deduce the following explicit description of the additive structure of the 2-torsion of $H^{*}\left(B S L\left(\mathbb{F}_{p}\right)^{+} ; \mathbb{Z}\right)$. Observe in particular that there is no direct summand of order 2 in $H^{*}\left(B S L\left(\mathbb{F}_{p}\right)^{+} ; \mathbb{Z}\right)$.

Theorem 3.7. Let $p$ be any prime $\equiv 5 \bmod 8$ and consider the graded $\mathbb{Z}$-algebra

$$
\Phi=\mathbb{Z}\left[a_{k}, k \geq 2\right] \otimes \Lambda_{\mathbb{Z}}\left(b_{k}, k \text { even } \geq 2\right),
$$

where $\operatorname{deg}\left(a_{k}\right)=2 k$ and $\operatorname{deg}\left(b_{k}\right)=2 k-1$.

As a graded abelian group, the 2-torsion subgroup of $H^{*}\left(B S L\left(\mathbb{F}_{p}\right)^{+} ; \mathbb{Z}\right)$ is additively isomorphic to the graded $\Phi$-module generated by

$$
\left\{H_{A, r} \mid r \geq 2, A \text { running over all non-empty finite subsets of } N_{r}\right\}
$$

with relations generated by

$$
\begin{cases}2^{r} H_{A, r}, & \text { for all } k \in N_{s} \text { with } 2 \leq s<r, \\ a_{k} H_{A, r} & \text { for all } k \in N_{s} \text { with } 2 \leq s \leq r, \\ b_{k} H_{A, r} & \text { for all } r \geq 2, A \subset N_{r} .\end{cases}
$$

The element $H_{\left\{k_{1}, \ldots, k_{t}\right\}, r} \in H^{*}\left(B S L\left(\mathbb{F}_{p}\right)^{+} ; \mathbb{Z}\right)$ is of degree $2\left(\sum_{j=1}^{t} k_{j}\right)-t+1$ and reduces mod 2 to the class $\sum_{j=1}^{t} c_{k_{j}} e_{k_{1}} \cdots e_{k_{(j-1)}} e_{k_{(j+1)}} \cdots e_{k_{t}} \in H^{*}\left(B S L\left(\mathbb{F}_{p}\right)^{+} ; \mathbb{Z} / 2\right)$.

Proof. Let us denote by $P_{r}$ the polynomial tensor factor $\mathbb{Z} / 2\left[c_{k}, 2 \leq k \in N_{r}\right]$ of $F_{r}$ for $r \geq 2$. According to Corollary 3.5, the image of $d_{r}$ is

$$
\operatorname{Im}\left(d_{r}\right)=\operatorname{Im}\left(\delta_{r}\right) \otimes\left(\bigotimes_{s>r} F_{s}\right)
$$

and Lemma 3.6 implies that $\operatorname{Im}\left(d_{r}\right)$ is the $\left(P_{r} \otimes\left(\bigotimes_{s>r} F_{s}\right)\right)$-module generated by the $H_{A, r}$ 's, where $A$ runs over all non-empty finite subsets of $N_{r}$, with the relations given by Lemma 3.6. By definition of $\Phi$, the generators $a_{k}$ and $b_{k}$ of the $\mathbb{Z}$-algebra $\Phi$ are in one-to-one correspondence with the classes $c_{k}$ and $e_{k}$ respectively, which generate the $\mathbb{Z} / 2$-algebra

$$
P_{2} \otimes\left(\bigotimes_{s>2} F_{s}\right) \cong \mathbb{Z} / 2\left[c_{k}, k \geq 2\right] \otimes \Lambda_{\mathbb{Z} / 2}\left(e_{k}, k \text { even } \geq 2\right) .
$$


The assertion then follows by gluing together the information on $\operatorname{Im}\left(d_{r}\right)$ for $r \geq 2$.

Remark 3.8. The additive structure of $H^{*}\left(B S L\left(\mathbb{F}_{p}\right)^{+} ; \mathbb{Z}\right)$ has been already calculated in $[\mathrm{Hu}]$, but in a completely different way.

\section{The $\bmod 2$ Bockstein spectral sequence for $B S L(\mathbb{Z})^{+}$}

Finally, let us investigate the mod 2 Bockstein spectral sequence

$$
E_{1}^{*}\left(B S L(\mathbb{Z})^{+}\right) \cong \mathbb{Z} / 2\left[w_{j}, j \geq 2\right] \otimes \Lambda_{\mathbb{Z} / 2}\left(u_{2 k-1}, k \geq 2\right) \Longrightarrow\left(H^{*}\left(B S L(\mathbb{Z})^{+} ; \mathbb{Z}\right) / \text { torsion }\right) \otimes \mathbb{Z} / 2
$$

for the mod 2 cohomology of the space $B S L(\mathbb{Z})^{+}$. Since the induced homomorphism $h^{*}: H^{*}(B S O ; \mathbb{Z} / 2) \rightarrow$ $H^{*}\left(B S L(\mathbb{Z})^{+} ; \mathbb{Z} / 2\right)$ sends the Stiefel-Whitney classes $w_{j} \in H^{j}(B S O ; \mathbb{Z} / 2)$ onto the corresponding classes $w_{j} \in H^{j}\left(B S L(\mathbb{Z})^{+} ; \mathbb{Z} / 2\right)$, we have again $S q^{1}\left(w_{2 i}\right)=w_{2 i+1}, S q^{1}\left(w_{2 i+1}\right)=0$ for $i \geq 1$ and we know from Lemma 12 of $[\mathrm{AMNY}]$ that $S q^{1}\left(u_{2 k-1}\right)=0$ for $k \geq 2$. Therefore, we obtain the $E_{2}$-term as follows:

$$
E_{2}^{*}\left(B S L(\mathbb{Z})^{+}\right) \cong E_{2}^{*}(B S O) \otimes \Lambda_{\mathbb{Z} / 2}\left(u_{2 k-1}, k \geq 2\right) \cong \mathbb{Z} / 2\left[w_{2 i}^{2}, i \geq 1\right] \otimes \Lambda_{\mathbb{Z} / 2}\left(u_{2 k-1}, k \geq 2\right) .
$$

Because of the naturality of the mod 2 Bockstein spectral sequence with respect to $h^{*}$, we may deduce from Section 1 that all higher differentials $d_{r}$ are trivial on $\mathbb{Z} / 2\left[w_{2 i}^{2}, i \geq 1\right], r \geq 2$.

Lemma 4.1. For all positive integers $r$ and $i$, one has $d_{r}\left(u_{4 i+1}\right)=0$ in $E_{r}^{4 i+2}\left(B S L(\mathbb{Z})^{+}\right)$.

Proof. This is true if $r=1$. For $r \geq 2, d_{r}\left(u_{4 i+1}\right)$ is of the form $d_{r}\left(u_{4 i+1}\right)=\sum_{s} w(s) \otimes u(s)$, where $w(s)$ is a product of classes $w_{2 i}^{2}(i \geq 1)$ and $u(s)$ a product of classes $u_{2 k-1}(k \geq 2)$. According to Proposition 7 of $[\mathrm{AMNY}]$, the classes $u_{2 k-1}(k \geq 2)$ are primitive cohomology classes, in other words, $\mu^{*}\left(u_{2 k-1}\right)=$ $u_{2 k-1} \otimes 1+1 \otimes u_{2 k-1}$, where $\mu^{*}$ is the coproduct $H^{*}\left(B S L(\mathbb{Z})^{+} ; \mathbb{Z} / 2\right) \rightarrow H^{*}\left(B S L(\mathbb{Z})^{+} \times B S L(\mathbb{Z})^{+} ; \mathbb{Z} / 2\right)$ provided by the H-space structure of $B S L(\mathbb{Z})^{+}$. In particular, it follows from the fact that $E_{r}^{*}\left(B S L(\mathbb{Z})^{+}\right)$ is a differential Hopf algebra (see Proposition 4.7 of $[\operatorname{Brd}])$ that $\mu^{*}\left(d_{r}\left(u_{4 i+1}\right)\right)=d_{r}\left(\mu^{*}\left(u_{4 i+1}\right)\right)$ and consequently that $d_{r}\left(u_{4 i+1}\right)$ is primitive. However, for $\sum_{s} w(s) \otimes u(s)$ to be primitive, it is necessary to have $u(s)$ primitive, which is only possible if $u(s)=1$ or $u(s)=u_{2 k-1}$ for some $k$. In both cases, the element $w(s) \otimes u(s)$ cannot lie in degree $4 i+2$ since $\operatorname{deg}(w(s)) \equiv 0 \bmod 4$. Consequently, the sum must be empty and we get $d_{r}\left(u_{4 i+1}\right)=0$.

Now, let us consider any prime $p \equiv 5 \bmod 8$ and the homomorphism

$$
f_{p}^{*}: H^{*}\left(B S L\left(\mathbb{F}_{p}\right)^{+} ; \mathbb{Z} / 2\right) \cong \mathbb{Z} / 2\left[c_{k}, k \geq 2\right] \otimes \Lambda_{\mathbb{Z} / 2}\left(e_{k}, k \geq 2\right) \longrightarrow H^{*}\left(B S L(\mathbb{Z})^{+} ; \mathbb{Z} / 2\right)
$$

induced by the reduction $\bmod p$. We shall replace the generators $u_{4 i-1}$ (for $i \geq 1$ ) of the exterior subalgebra $\Lambda_{\mathbb{Z} / 2}\left(u_{2 k-1}, k \geq 2\right)$ of $H^{*}\left(B S L(\mathbb{Z})^{+} ; \mathbb{Z} / 2\right)$ by

$$
\varepsilon_{4 i-1}=f_{p}^{*}\left(e_{2 i}\right)=u_{4 i-1}+\sum_{j=2}^{2 i-2} w_{j}^{2} u_{4 i-2 j-1} \in H^{4 i-1}\left(B S L(\mathbb{Z})^{+} ; \mathbb{Z} / 2\right)
$$

(see Theorem 13 of $[\mathrm{AMNY}])$. Thus, the first two terms of the mod 2 Bockstein spectral sequence of $B S L(\mathbb{Z})^{+}$ can be expressed as

$$
\begin{gathered}
E_{1}^{*}\left(B S L(\mathbb{Z})^{+}\right) \cong H^{*}\left(B S L(\mathbb{Z})^{+} ; \mathbb{Z} / 2\right) \cong \mathbb{Z} / 2\left[w_{j}, j \geq 2\right] \otimes \Lambda_{\mathbb{Z} / 2}\left(\varepsilon_{4 i-1}, i \geq 1\right) \otimes \Lambda_{\mathbb{Z} / 2}\left(u_{4 i+1}, i \geq 1\right) \\
E_{2}^{*}\left(B S L(\mathbb{Z})^{+}\right) \cong \mathbb{Z} / 2\left[w_{2 i}^{2}, i \geq 1\right] \otimes \Lambda_{\mathbb{Z} / 2}\left(\varepsilon_{4 i-1}, i \geq 1\right) \otimes \Lambda_{\mathbb{Z} / 2}\left(u_{4 i+1}, i \geq 1\right)
\end{gathered}
$$

Let us consider again the sets of integers $N_{r}=\left\{k=2^{r-1} i+2^{r-2} \mid i \geq 0\right\}$ introduced in Definition 3.1. 
Lemma 4.2. For any $r \geq 3$, if $k \in N_{r}$, then $\varepsilon_{2 k-1}$ belongs to $E_{s}^{*}\left(B S L(\mathbb{Z})^{+}\right)$for all $s \leq r$ and $d_{r}\left(\varepsilon_{2 k-1}\right)=w_{k}^{2}$.

Proof. By naturality of the mod 2 Bockstein spectral sequence with respect to $f_{p}^{*}$, this follows from the equality

$$
d_{r}\left(e_{k}\right)=c_{k} \text { for } k \in N_{r}
$$

given by Lemma 3.3, from the definition $\varepsilon_{2 k-1}=f_{p}^{*}\left(e_{k}\right)$ (where $k$ is even since $k \in N_{r}$ with $r \geq 3$ ) and the formula $f_{p}^{*}\left(c_{k}\right)=w_{k}^{2}$ (see Lemma 1.4 of $\left.[\operatorname{Ar} 3]\right)$.

This argument implies also the vanishing of $d_{2}$, because $w_{k}^{2}=0$ in $E_{2}^{*}\left(B S L(\mathbb{Z})^{+}\right)$when $k$ belongs to $N_{2}=$ odd positive integers $\}$. Let us summarize the information we obtain on $E_{r}^{*}\left(B S L(\mathbb{Z})^{+}\right)$.

Theorem 4.3. The mod 2 Bockstein spectral sequence for $B S L(\mathbb{Z})^{+}$satisfies:

(a) $E_{1}^{*}\left(B S L(\mathbb{Z})^{+}\right) \cong H^{*}\left(B S L(\mathbb{Z})^{+} ; \mathbb{Z} / 2\right) \cong \mathbb{Z} / 2\left[w_{j}, j \geq 2\right] \otimes \Lambda_{\mathbb{Z} / 2}\left(\varepsilon_{4 i-1}, i \geq 1\right) \otimes \Lambda_{\mathbb{Z} / 2}\left(u_{4 i+1}, i \geq 1\right)$ and $d_{1}\left(w_{2 i}\right)=w_{2 i+1}, d_{1}\left(w_{2 i+1}\right)=0$, and $d_{1}$ is trivial on all classes $\varepsilon_{4 i-1}$ and $u_{4 i+1} \quad(i \geq 1)$.

(b) $E_{2}^{*}\left(B S L(\mathbb{Z})^{+}\right) \cong E_{3}^{*}\left(B S L(\mathbb{Z})^{+}\right) \cong \mathbb{Z} / 2\left[w_{k}^{2}, k\right.$ even $\left.\geq 2\right] \otimes \Lambda_{\mathbb{Z} / 2}\left(\varepsilon_{2 k-1}, k\right.$ even $\left.\geq 2\right) \otimes \Lambda_{\mathbb{Z} / 2}\left(u_{4 i+1}, i \geq 1\right)$.

(c) For any $r \geq 3$,

$$
E_{r}^{*}\left(B S L(\mathbb{Z})^{+}\right) \cong \mathbb{Z} / 2\left[w_{k}^{2}, k \in N_{s} \text { for } s \geq r\right] \otimes \Lambda_{\mathbb{Z} / 2}\left(\varepsilon_{2 k-1}, k \in N_{s} \text { for } s \geq r\right) \otimes \Lambda_{\mathbb{Z} / 2}\left(u_{4 i+1}, i \geq 1\right)
$$

and $d_{r}\left(\varepsilon_{2 k-1}\right)=w_{k}^{2}$ whenever $k \in N_{r}$.

(d) $E_{\infty}^{*}\left(B S L(\mathbb{Z})^{+}\right) \cong \Lambda_{\mathbb{Z} / 2}\left(u_{4 i+1}, i \geq 1\right)$.

The calculation of the differentials in that mod 2 Bockstein spectral sequence enables us to split its $E_{1}$-term as a tensor product of differential graded $\mathbb{Z} / 2$-algebras:

$$
E_{1}^{*}\left(B S L(\mathbb{Z})^{+}\right) \cong D_{1} \otimes\left(\bigotimes_{s \geq 3} D_{s}\right) \otimes D_{\infty}
$$

where

$$
\begin{gathered}
D_{1}=\mathbb{Z} / 2\left[w_{2 i+1}, i \geq 1\right] \otimes \Lambda_{\mathbb{Z} / 2}\left(w_{2 i}, i \geq 1\right), \quad \delta_{1}\left(w_{2 i}\right)=w_{2 i+1}, \\
D_{s}=\mathbb{Z} / 2\left[w_{k}^{2}, k \in N_{s}\right] \otimes \Lambda_{\mathbb{Z} / 2}\left(\varepsilon_{2 k-1}, k \in N_{s}\right), \quad \delta_{s}\left(\varepsilon_{2 k-1}\right)=w_{k}^{2}, \quad \text { for } s \geq 3, \\
D_{\infty}=\Lambda_{\mathbb{Z} / 2}\left(u_{4 i+1}, i \geq 1\right), \quad \delta_{\infty}=0 .
\end{gathered}
$$

The spectral sequence can then be described in the following simple way.

Corollary 4.4. The mod 2 Bockstein spectral sequence for $B S L(\mathbb{Z})^{+}$satisfies:

(a) $E_{1}^{*}\left(B S L(\mathbb{Z})^{+}\right) \cong D_{1} \otimes\left(\bigotimes_{s \geq 3} D_{s}\right) \otimes D_{\infty}$ and the first differential is $d_{1}=\delta_{1}$ on $D_{1}$ and $d_{1}=0$ on $D_{s}$ when $3 \leq s \leq \infty$.

(b) $E_{2}^{*}\left(B S L(\mathbb{Z})^{+}\right) \cong E_{3}^{*}\left(B S L(\mathbb{Z})^{+}\right)$and for $r \geq 3, E_{r}^{*}\left(B S L(\mathbb{Z})^{+}\right) \cong\left(\bigotimes_{s \geq r} D_{s}\right) \otimes D_{\infty}$ with the differential $d_{r}=\delta_{r}$ on $D_{r}$ and $d_{r}=0$ on $D_{s}$ when $r<s \leq \infty$.

(d) $E_{\infty}^{*}\left(B S L(\mathbb{Z})^{+}\right) \cong D_{\infty}$.

Remark 4.5. Let us mention that the mod 2 Bockstein spectral sequence for the group $S L_{3}\left(\mathbb{Z}\left[\frac{1}{2}\right]\right)$ has been recently computed by H.-W. Henn in Section 4.3 of [He].

The following interesting observations are immediate consequences of Theorem 4.3 (b) and (d) and Corollary 4.4 (b) and (d). 


\section{Corollary 4.6.}

(a) There is no cyclic direct summand of order 4 in $H^{*}\left(B S L(\mathbb{Z})^{+} ; \mathbb{Z}\right)$.

(b) The set of all non-trivial elements of $\Lambda_{\mathbb{Z} / 2}\left(u_{4 i+1}, i \geq 1\right)$ is in one-to-one correspondence with an additive basis of $H^{*}\left(B S L(\mathbb{Z})^{+} ; \mathbb{Z}\right) /$ torsion .

By applying again Lemma 3.6, we get an explicit description of the additive structure of the 2-torsion of the integral cohomology $H^{*}\left(B S L(\mathbb{Z})^{+} ; \mathbb{Z}\right)$. In order to formulate the main result of this section, let us use again the notation introduced in Remark 2.2 and Definition 3.1: $N_{1}=\mathbb{N}-\{0\}$ and $N_{r}=\left\{k=2^{r-1} i+2^{r-2} \mid i \geq 0\right\}$ for $r \geq 3$.

Theorem 4.7. Consider the graded $\mathbb{Z}$-algebra

$$
\Omega=\mathbb{Z}\left[\omega_{k, 1}, k \in N_{1}\right] \otimes \mathbb{Z}\left[\omega_{k, r}, k \in N_{r} \text { with } r \geq 3\right] \otimes \Lambda_{\mathbb{Z}}\left(z_{2 k-1}, k \geq 2\right),
$$

where $\operatorname{deg}\left(\omega_{k, 1}\right)=2 k+1, \operatorname{deg}\left(\omega_{k, r}\right)=2 k$ when $r \geq 3$ and $\operatorname{deg}\left(z_{2 k-1}\right)=2 k-1$.

As a graded abelian group, the 2-torsion subgroup of $H^{*}\left(B S L(\mathbb{Z})^{+} ; \mathbb{Z}\right)$ is additively isomorphic to the graded $\Omega$-module generated by

$$
\left\{J_{A, r} \mid r=1 \text { or } r \geq 3, A \text { running over all non-empty finite subsets of } N_{r}\right\}
$$

with relations generated by

$$
\begin{cases}2^{r} J_{A, r}, & \text { for all } s<r \text { and all } k \in N_{s}, \text { when } r \geq 3, \\ \omega_{k, s} J_{A, r} & \text { for all } k \in N_{s} \text { with } 3 \leq s \leq r, \text { when } r \geq 3, \\ z_{2 k-1} J_{A, r} & \text { for } r=1 \text { and } r \geq 3, A \subset N_{r} . \\ \sum_{k \in A} \omega_{k, r} J_{A-\{k\}, r}\end{cases}
$$

The element $J_{\left\{k_{1}, \ldots, k_{t}\right\}, 1} \in H^{*}\left(B S L(\mathbb{Z})^{+} ; \mathbb{Z}\right)$ is of degree $2\left(\sum_{j=1}^{t} k_{j}\right)+1$ and reduces mod 2 to the class $\sum_{j=1}^{t} w_{2 k_{j}+1} w_{2 k_{1}} \cdots w_{2 k_{(j-1)}} w_{2 k_{(j+1)}} \cdots w_{2 k_{t}} \in H^{*}\left(B S L(\mathbb{Z})^{+} ; \mathbb{Z} / 2\right)$. For $r \geq 3$, the element $J_{\left\{k_{1}, \ldots, k_{t}\right\}, r} \in H^{*}\left(B S L(\mathbb{Z})^{+} ; \mathbb{Z}\right)$ is of degree $2\left(\sum_{j=1}^{t} k_{j}\right)-t+1$ and reduces mod 2 to the class $\sum_{j=1}^{t} w_{k_{j}}^{2} \varepsilon_{2 k_{1}-1} \cdots \varepsilon_{2 k_{(j-1)}-1} \varepsilon_{2 k_{(j+1)}-1} \cdots \varepsilon_{2 k_{t}-1} \in H^{*}\left(B S L(\mathbb{Z})^{+} ; \mathbb{Z} / 2\right)$.

Proof. For $r=1$ or $r \geq 3$, let us call $P_{r}$ the polynomial tensor factor of $D_{r}$. Because of the splitting of $E_{r}^{*}\left(B S L(\mathbb{Z})^{+}\right)$given by Corollary 4.4, the image of $d_{r}$ is

$$
\operatorname{Im}\left(d_{r}\right)=\operatorname{Im}\left(\delta_{r}\right) \otimes\left(\bigotimes_{s>r} D_{s}\right) \otimes D_{\infty}
$$

and Lemma 3.6 implies that $\operatorname{Im}\left(d_{r}\right)$ is the $\left(P_{r} \otimes\left(\bigotimes_{s>r} D_{s}\right) \otimes D_{\infty}\right)$-module generated by the $J_{A, r}$ 's, where $A$ runs over all non-empty finite subsets of $N_{r}$, with the relations provided by Lemma 3.6. Now, let us denote by $\Omega$ the graded $\mathbb{Z}$-algebra $\Omega=\mathbb{Z}\left[\omega_{k, 1}, k \in N_{1}\right] \otimes \mathbb{Z}\left[\omega_{k, r}, k \in N_{r}\right.$ with $\left.r \geq 3\right] \otimes \Lambda_{\mathbb{Z}}\left(z_{2 k-1}, k \geq 2\right)$ whose generators are in one-to-one correspondence with those of

$$
\begin{aligned}
& P_{1} \otimes\left(\bigotimes_{s \geq 3} D_{s}\right) \otimes D_{\infty} \cong \\
& \mathbb{Z} / 2\left[w_{2 k+1}, k \geq 1\right] \otimes \mathbb{Z} / 2\left[w_{k}^{2}, k \text { even } \geq 2\right] \otimes \Lambda_{\mathbb{Z} / 2}\left(\varepsilon_{2 k-1}, k \text { even } \geq 2\right) \otimes \Lambda_{\mathbb{Z} / 2}\left(u_{2 k-1}, k \text { odd } \geq 3\right)
\end{aligned}
$$

as follows: $\omega_{k, 1}$ corresponds to $w_{2 k+1}, \omega_{k, r}$ to $w_{k}^{2}$ when $r \geq 3$ and $k \in N_{r}, z_{2 k-1}$ to $\varepsilon_{2 k-1}$ when $k$ is even and to $u_{2 k-1}$ when $k$ is odd. The assertion of the theorem then follows by gluing together the information on the elements of order $2^{r}$ in $H^{*}\left(B S L(\mathbb{Z})^{+} ; \mathbb{Z}\right)$ given by the determination of $\operatorname{Im}\left(d_{r}\right)$ for $r \geq 1$.

Remark 4.8. The isomorphism established in Theorem 4.7 is an additive isomorphism: for instance, for $3 \leq s<r, k \in N_{s}$ and $A$ a non-empty finite subset of $N_{r}$, the product of the elements of $H^{*}\left(B S L(\mathbb{Z})^{+} ; \mathbb{Z}\right)$ 
corresponding to $J_{\{k\}, s}$ and $J_{A, r}$ under that isomorphism is non-trivial, even if the reduction mod 2 of $J_{\{k\}, s}$ is $w_{k}^{2}$, which is the generator of $D_{s}$ corresponding to the generator $\omega_{k, s}$ of $\Omega$ (that product is actually an element of order $2^{s}$ in $H^{*}\left(B S L(\mathbb{Z})^{+} ; \mathbb{Z}\right)$ which reduces mod 2 to the reduction $\bmod 2$ of $\left(\sum_{k \in A} \omega_{k, r} \prod_{i \in A-\{k\}} z_{2 i-1}\right) J_{\{k\}, s}$, where $\left.\left(\sum_{k \in A} \omega_{k, r} \prod_{i \in A-\{k\}} z_{2 i-1}\right) \in \Omega\right)$. More generally, the above mod 2 Bockstein spectral sequence calculation provides the following multiplicative relations mod 2 between the additive generators of the 2-torsion subgroup of $H^{*}\left(B S L(\mathbb{Z})^{+} ; \mathbb{Z}\right)$ given by Theorem 4.7.

If $1 \leq s<r$, then it is obvious that

$$
J_{A, r} J_{B, s} \equiv\left(\sum_{k \in A} \omega_{k, r} \prod_{i \in A-\{k\}} z_{2 i-1}\right) J_{B, s} \quad \bmod 2 .
$$

If $r \geq 3$ and if $\operatorname{red}_{2}$ denotes again the reduction mod 2 , one has

$$
\begin{aligned}
\operatorname{red}_{2}\left(J_{A, r} J_{B, r}\right) & =\operatorname{red}_{2}\left(J_{A, r}\right) \delta_{r}\left(\prod_{j \in B} \varepsilon_{2 j-1}\right) \\
& =\delta_{r}\left(\operatorname{red}_{2}\left(J_{A, r}\right) \prod_{j \in B} \varepsilon_{2 j-1}\right) \\
& =\delta_{r}\left(\sum_{k \in A} w_{k}^{2} \prod_{i \in A-\{k\}} \varepsilon_{2 i-1} \prod_{j \in B} \varepsilon_{2 j-1}\right) .
\end{aligned}
$$

The fact that the classes $\varepsilon_{2 i-1}$ are exterior implies that

$$
\operatorname{red}_{2}\left(J_{A, r} J_{B, r}\right)=\delta_{r}\left(\sum_{\substack{k \in A \text { such that } \\ A-\{k\} \cap B=\emptyset}} w_{k}^{2} \prod_{i \in A-\{k\} \cup B} \varepsilon_{2 i-1}\right)=\sum_{\substack{k \in A \text { such that } \\ A-\{k\} \cap B=\emptyset}} w_{k}^{2} \delta_{r}\left(\prod_{i \in A-\{k\} \cup B} \varepsilon_{2 i-1}\right)
$$

and finally that

$$
J_{A, r} J_{B, r} \equiv \sum_{\substack{k \in A \text { such that } \\ A-\{k\} \cap B=\emptyset}} \omega_{k, r} J_{A-\{k\} \cup B, r} \bmod 2 .
$$

If $r=1$, the formula

$$
J_{A, 1} J_{B, 1} \equiv \sum_{\substack{k \in A \text { such that } \\ A-\{k\} \cap B=\emptyset}} \omega_{k, 1} J_{A-\{k\} \cup B, 1} \bmod 2
$$

does still hold but the classes $\varepsilon_{2 i-1}$ should be replaced by $w_{2 i}$ in the argument.

Finally, the above computation helps us to understand, at the prime 2 , the homomorphisms $h^{*}: H^{*}(B S O ; \mathbb{Z}) \rightarrow H^{*}\left(B S L(\mathbb{Z})^{+} ; \mathbb{Z}\right)$ and $f_{p}^{*}: H^{*}\left(B S L\left(\mathbb{F}_{p}\right) ; \mathbb{Z}\right) \rightarrow H^{*}\left(B S L(\mathbb{Z})^{+} ; \mathbb{Z}\right)$ induced by the inclusion $\mathbb{Z} \hookrightarrow \mathbb{R}$ and by the reduction $\bmod p$, when $p \equiv 5 \bmod 8$.

\section{Theorem 4.9.}

(a) The homomorphism $h^{*}: H^{*}(B S O ; \mathbb{Z}) \rightarrow H^{*}\left(B S L(\mathbb{Z})^{+} ; \mathbb{Z}\right)$ is injective on the torsion classes of $H^{*}(B S O ; \mathbb{Z})$.

(b) For every generator of infinite order $p_{2 k}$ in $H^{2 k}(B S O ; \mathbb{Z})$ with $k$ even, $h^{*}\left(p_{2 k}\right)$ is an element of order $2^{r}$ if $k$ belongs to $N_{r}, r \geq 3$ (up to odd torsion).

(c) For $p \equiv 5 \bmod 8$, the image of any generator of any cyclic direct summand of order 4 in $H^{*}\left(B S L\left(\mathbb{F}_{p}\right)^{+} ; \mathbb{Z}\right)$ under the homomorphism $f_{p}^{*}: H^{*}\left(B S L\left(\mathbb{F}_{p}\right)^{+} ; \mathbb{Z}\right) \rightarrow H^{*}\left(B S L(\mathbb{Z})^{+} ; \mathbb{Z}\right)$ has order 2 in $H^{*}\left(B S L(\mathbb{Z})^{+} ; \mathbb{Z}\right)$.

(d) For any $r \geq 3$, the homomorphism $f_{p}^{*}$ (with $p \equiv 5 \bmod 8$ ) is injective on all cyclic direct summands of order $2^{r}$ in $H^{*}\left(B S L\left(\mathbb{F}_{p}\right)^{+} ; \mathbb{Z}\right)$. 
Proof. Assertion (a) is obvious since the Stiefel-Whitney classes $w_{j} \in H^{j}(B S O ; \mathbb{Z})$ correspond to the elements $w_{j} \in H^{j}\left(B S L(\mathbb{Z})^{+} ; \mathbb{Z}\right)$ via $h^{*}$. The fact that the reduction $\bmod 2$ of $p_{2 k}$ is $w_{k}^{2} \in H^{2 k}(B S O ; \mathbb{Z} / 2)$, by Proposition 2.1 (c), and that $d_{r}\left(\varepsilon_{2 k-1}\right)=w_{k}^{2}$ if $k \in N_{r}$, according to Theorem 4.3 (c), implies (b). According to Theorem 3.7, the generators of the cyclic direct summands of order $2^{r}$ in $H^{*}\left(B S L\left(\mathbb{F}_{p}\right)^{+} ; \mathbb{Z}\right)$ belong to the $\Phi$-module generated by the $H_{A, r}$ 's, where $A=\left\{k_{1}, \ldots, k_{t}\right\}$ is a finite subset of $N_{r}$ and one has:

$$
f_{p}^{*}\left(\operatorname{red}_{2}\left(H_{A, r}\right)\right)=f_{p}^{*}\left(\sum_{j=1}^{t} c_{k_{j}} e_{k_{1}} \cdots e_{k_{(j-1)}} e_{k_{(j+1)}} \cdots e_{k_{t}}\right)=\sum_{j=1}^{t} w_{k_{j}}^{2} \varepsilon_{2 k_{1}-1} \cdots \varepsilon_{2 k_{(j-1)}-1} \varepsilon_{2 k_{(j+1)}-1} \cdots \varepsilon_{2 k_{t}-1}
$$

since $f_{p}^{*}\left(c_{k_{j}}\right)=w_{k_{j}}^{2}$ by Lemma 1.4 of [Ar3]. If $r=2, N_{2}=\{$ odd positive integers $\}$ and Assertion (c) follows from the equality

$$
d_{1}\left(w_{k_{j}-1} w_{k_{j}} \varepsilon_{2 k_{1}-1} \cdots \varepsilon_{2 k_{(j-1)}-1} \varepsilon_{2 k_{(j+1)}-1} \cdots \varepsilon_{2 k_{t}-1}\right)=w_{k_{j}}^{2} \varepsilon_{2 k_{1}-1} \cdots \varepsilon_{2 k_{(j-1)}-1} \varepsilon_{2 k_{(j+1)}-1} \cdots \varepsilon_{2 k_{t}-1}
$$

when $k_{j}$ is odd. If $r \geq 3$, then Assertion (d) is a consequence of

$$
d_{r}\left(\prod_{j=1}^{t} \varepsilon_{2 k_{j}-1}\right)=\sum_{j=1}^{t} w_{k_{j}}^{2} \varepsilon_{2 k_{1}-1} \cdots \varepsilon_{2 k_{(j-1)}-1} \varepsilon_{2 k_{(j+1)}-1} \cdots \varepsilon_{2 k_{t}-1}
$$

in $E_{r}^{*}\left(B S L(\mathbb{Z})^{+}\right)$.

\section{Chern classes of integral representations of groups}

For $n \geq 1$, let us call $c_{n}(S L(\mathbb{Z})) \in H^{2 n}(S L(\mathbb{Z}) ; \mathbb{Z})$ the $n$-th Chern class of the inclusion $\sigma: S L(\mathbb{Z}) \hookrightarrow$ $G L(\mathbb{C})$, i.e., $\quad c_{n}(S L(\mathbb{Z}))=\sigma^{*}\left(\bar{c}_{n}\right)$, where $\bar{c}_{n}$ denotes the $n$-th universal Chern class of degree $2 n$ in $H^{*}(B G L(\mathbb{C}) ; \mathbb{Z}) \cong \mathbb{Z}\left[\bar{c}_{1}, \bar{c}_{2}, \ldots, \bar{c}_{n}, \ldots\right]$ and $\sigma^{*}: H^{*}(B G L(\mathbb{C}) ; \mathbb{Z}) \rightarrow H^{*}(B S L(\mathbb{Z}) ; \mathbb{Z}) \cong H^{*}(S L(\mathbb{Z}) ; \mathbb{Z})$ the homomorphism induced by $\sigma$. The order of $c_{n}(S L(\mathbb{Z}))$ has been only determined up to a factor 2 .

Definition 5.1. For any positive even integer $n$, let $E_{n}$ be the denominator of $\frac{B_{n}}{n}$, where $B_{n}$ is the $n$-th Bernoulli number; for instance, $B_{2}=\frac{1}{6}, B_{4}=-\frac{1}{30}, B_{6}=\frac{1}{42}$, and $E_{2}=12, E_{4}=120, E_{6}=252$.

Proposition 5.2. The Chern classes $c_{n}(S L(\mathbb{Z}))$ are all torsion classes. If $n$ is odd, then $c_{n}(S L(\mathbb{Z}))$ is of order 2 in $H^{2 n}(S L(\mathbb{Z}) ; \mathbb{Z})$. If $n$ is even, then the order of $c_{n}(S L(\mathbb{Z}))$ in $H^{2 n}(S L(\mathbb{Z}) ; \mathbb{Z})$ is equal to $2 E_{n}$ when $n \equiv 2 \bmod 4$, and to $E_{n}$ or $2 E_{n}$ when $n \equiv 0 \bmod 4$.

Proof. See [EM1], Section 5, [EM2], Main Theorem, and [Ar1], Einleitung and Korollar 2.5.

The determination of the exact order of $c_{n}(S L(\mathbb{Z}))$ in the case where $n \equiv 0 \bmod 4$ now follows from the mod 2 Bockstein spectral sequence calculations presented in Theorem 4.3 and Corollary 4.4.

Theorem 5.3. For any positive even integer $n$, the Chern class $c_{n}(S L(\mathbb{Z}))$ is a torsion class of order $2 E_{n}$ in $H^{2 n}(S L(\mathbb{Z}) ; \mathbb{Z})$.

Proof. The odd-primary part of the order of $c_{n}(S L(\mathbb{Z}))$ is given by Proposition 5.2. For all integers $r \geq 3$, we know from Lemma 4.2 that $d_{r}\left(\varepsilon_{2 n-1}\right)=w_{n}^{2}$ when $n \in N_{r}$. Therefore, the fact that $\operatorname{red}_{2}\left(c_{n}(S L(\mathbb{Z}))\right)=w_{n}^{2}$ for all $n \geq 1$ (see [MT], Part I, p.137, Theorem 5.11) implies that for $n$ even, $c_{n}\left(S L(\mathbb{Z})\right.$ ) is of order $2^{r}$ in $H^{n}(S L(\mathbb{Z}) ; \mathbb{Z})$ when $n \in N_{r} \quad(r \geq 3)$. On the other hand, according to von Staudt's theorem (see [BS], p.384, Theorem 4$), 2^{t}$ divides $E_{n}$ if and only if $2^{t-1}$ divides $n$. Thus, if $n \in N_{r}=\left\{n \in \mathbb{N} \mid v_{2}(n)=r-2\right\}$, 
then the 2-primary part of $E_{n}$ is $2^{r-1}$. Consequently, the 2-primary parts of the order of $c_{n}(S L(\mathbb{Z}))$ and of the integer $2 E_{n}$ coincide for any even integer $n \geq 2$.

Remark 5.4. The same result holds for the Chern classes $c_{n}(G L(\mathbb{Z}))$ of the general linear group $G L(\mathbb{Z})$ since there is a homotopy equivalence $B G L(\mathbb{Z})^{+} \simeq B S L(\mathbb{Z})^{+} \times B \mathbb{Z} / 2$ (see for instance [Ar1], Lemma 1.2).

The knowledge of the order of the Chern classes of $S L(\mathbb{Z})$ produces the following result on the Chern classes of the linear groups over the field of rationals $\mathbb{Q}$.

Corollary 5.5. The Chern classes $c_{n}(S L(\mathbb{Q}))$ and $c_{n}(G L(\mathbb{Q}))$ are all torsion classes. If $n$ is odd, they are of order 2. If $n$ is even, the order of $c_{n}(S L(\mathbb{Q}))$ and of $c_{n}(G L(\mathbb{Q}))$ is equal to $2 E_{n}$.

Proof. Since the order of $c_{n}(S L(\mathbb{Q}))$ and of $c_{n}(G L(\mathbb{Q}))$ is a positive multiple of the order of $c_{n}(S L(\mathbb{Z}))$, a lower bound for it is given by Proposition 5.2 and Theorem 5.3. The assertion then follows from Theorem 11 of $[\operatorname{Ar} 2]$.

For any complex representation $\rho: G \rightarrow G L(\mathbb{C})$ of any discrete group $G$, the Chern classes of $\rho$ are $c_{n}(\rho)=\rho^{*}\left(\bar{c}_{n}\right) \in H^{2 n}(G ; \mathbb{Z})$, where $\rho^{*}$ is the induced homomorphism $H^{2 n}(B G L(\mathbb{C}) ; \mathbb{Z}) \rightarrow H^{2 n}(B G ; \mathbb{Z}) \cong$ $H^{2 n}(G ; \mathbb{Z})$. Of course, the above calculations produce the following consequence for any integral representation $\rho: G \rightarrow G L(\mathbb{Z}) \hookrightarrow G L(\mathbb{C})$ or any rational representation $\rho: G \rightarrow G L(\mathbb{Q}) \hookrightarrow G L(\mathbb{C})$ of any discrete group $G$.

Corollary 5.6. The best upper bound for the order of the $n$-th Chern class $c_{n}(\rho)$ of any integral or rational representation $\rho$ of any discrete group $G$ is equal to 2 when $n$ is odd and to $2 E_{n}$ when $n$ is even.

Proof. Since $\rho$ is an integral representation, respectively a rational representation, $c_{n}(\rho)$ is the image of $c_{n}(G L(R))$ under the induced homomorphism $H^{2 n}(G L(R) ; \mathbb{Z}) \rightarrow H^{2 n}(G ; \mathbb{Z})$, where $R=\mathbb{Z}$, respectively $R=\mathbb{Q}$. Consequently, the order of $c_{n}(\rho)$ divides the order of $c_{n}(G L(R))$ which has been obtained in Proposition 5.2, Theorem 5.3, Remark 5.4 and Corollary 5.5. It turns out that the order of $c_{n}(S L(\mathbb{Z}))$ is the best possible upper bound since one can choose $G=S L(\mathbb{Z})$ and $\rho$ the inclusion into $G L(\mathbb{C})$.

Remark 5.7. The assertion of Corollary 5.6 is of particular interest because the best upper bound for the order of the $n$-th Chern class of any integral representation of any finite group is smaller, i.e., only equal to $E_{n}$ (see [EM1], Theorem 4.12, and [ThC], p. 89).

Acknowledgements. The third and fourth authors would like to thank the Swiss National Science Foundation for financial support when they visited the University of Lausanne and the JAMI of the Johns Hopkins University for its hospitality during the last stage of writing the manuscript. 


\section{References}

[Ar1] D. Arlettaz. Chern-Klassen von ganzzahligen und rationalen Darstellungen diskreter Gruppen. Math. Z. 187 (1984), 49-60.

[Ar2] D. Arlettaz. On the homology of the special linear group over a number fields. Comment. Math. Helv. 61 (1986), 556-564.

[Ar3] D. Arlettaz. Torsion classes in the cohomology of congruence subgroups. Math. Proc. Cambridge Philos. Soc. 105 (1989), 241-248.

[Ar4] D. Arlettaz. A note on the mod 2 cohomology of $S L(\mathbb{Z})$. In: Algebraic Topology Poznań 1989, Proceedings, Lecture Notes in Math. 1474 (1991), 365-370.

[AMNY] D. Arlettaz, M. Mimura, K. Nakahata and N. Yagita. The mod 2 cohomology of the linear groups over the ring of integers. Proc. Amer. Math. Soc. 127 (1999), 2199-2212.

[Au] C. Ausoni. Propriétés homotopiques de la K-théorie algébrique des entiers. Ph.D. thesis, Université de Lausanne (1998).

[Bo] A. Borel. Topics in the homology theory of fibre bundles. Lecture Notes in Math. 36 (Springer 1967).

[BS] Z. Borevich and I. Shafarevich. Number Theory (Academic Press 1973).

[Brd] W. Browder. Torsion in H-spaces. Ann. of Math. 74 (1961), 24-51.

[Brn] E. Brown. The cohomology of $B S O_{n}$ and $B O_{n}$ with integer coefficients. Proc. Amer. Math. Soc. 85 (1982), 283-288.

[CV] M. Čadek and J. Vanžura. The cohomology rings of $B O(n)$ and $B S O(n)$ with $\mathbb{Z}_{2^{m}}$ coefficients. J. Math. Kyoto Univ. 35 (1995), 35-41.

[EM1] B. Eckmann and G. Mislin. Chern classes of group representations over a number field. Compositio Math. 44 (1981), 41-65.

[EM2] B. Eckmann and G. Mislin. Profinite Chern classes of group representations. In: Topological topics, London Math. Soc. Lecture Note Ser. 86 (1983), 103-119.

[F] M. Feshbach. The integral cohomology rings of the classifying spaces of $O(n)$ and $S O(n)$. Indiana Univ. Math. J. 32 (1983), 511-516.

[He] H.-W. Henn. The cohomology of $S L(3, \mathbb{Z}[1 / 2])$. K-Theory 16 (1999), 299-359.

[Hu] J. Huebschmann. The cohomology of $F \Psi^{p}$. The additive structure. J. Pure Appl. Algebra 45 (1987), 73-91.

[MT] M. Mimura and H. Toda. Topology of Lie groups I and II. Translations of Math. Monographs 91 (AMS 1991).

[Q] D. Quillen. On the cohomology and K-theory of the general linear groups over a finite field. Ann. of Math. 96 (1972), 552-586.

[ThC] C. Thomas. Characteristic classes and the cohomology of finite groups. Cambridge Stud. Adv. Math. 9 (Cambridge University Press 1986).

[ThE] E. Thomas. On the cohomology of the real Grassmann complexes and the characteristic classes of n-plane bundles. Trans. Amer. Math. Soc. 96 (1960), 67-89. 